\title{
An asymptotic expansion related to the Dickman function
}

\author{
K. Soundararajan
}

In a recent paper Broadhurst [1] considered a generalized class of polylogarithms related to the Dickman function. In exploring these polylogarithms he was led to define a sequence of constants $C_{k}$ which he called the Dickman constants. Based on numerical computations, Broadhurst conjectured that the generating function $\sum_{k=0}^{\infty} C_{k} z^{k}$ equals $\exp (\gamma z) / \Gamma(1-z)$, so that the constants $C_{k}$ are related to values of the Riemann zeta-function at integers. In this note we establish Broadhurst's conjecture.

First we recall that the Dickman function $\rho:[0, \infty) \rightarrow[0,1]$ is defined by $\rho(u)=1$ for $0 \leq u \leq 1$, and for $u \geq 1$ is given by the unique solution to the differential difference equation $u \rho^{\prime}(u)=-\rho(u-1)$. The Dickman function arises naturally in number theory as follows: the number of integers below $x$ all of whose prime factors are below $x^{1 / u}$ is asymptotic to $\rho(u) x$ as $x \rightarrow \infty$. It is not hard to show that

$$
\rho(u)=\sum_{k=0}^{\infty} \frac{(-1)^{k}}{k !} I_{k}(u),
$$

where

$$
I_{k}(u)=\int_{\substack{t_{1}, \ldots t_{k} \geq 1 \\ t_{1}+\ldots+t_{k} \leq u}} \frac{d t_{1}}{t_{1}} \cdots \frac{d t_{k}}{t_{k}} .
$$

Note that $I_{0}(u)=1$ for all $u, I_{1}(u)=0$ for $0 \leq u \leq 1$ and $I_{1}(u)=\log u$ for $1 \leq u$, and so on. The sum over $k$ in the formula for $\rho(u)$ is in fact a finite sum since $I_{k}(u)=0$ if $k \geq u$.

Broadhurst uses a different formulation. He sets $F(\alpha)=\rho(1 / \alpha)$ which then satisfies $F(\alpha)=1$ for $\alpha \geq 1$, and for $0<\alpha<1$ satisfies the differential equation

$$
F^{\prime}(\alpha)=\frac{1}{\alpha} F\left(\frac{\alpha}{1-\alpha}\right) .
$$

He writes $F(\alpha)=\sum_{k=0}^{\infty} L_{k}(\alpha)$ where $L_{0}(\alpha)=1$ for all $\alpha$, and the "Dickman polylogarithm" $L_{k}$ is defined recursively by $L_{k}(t)=0$ for $t \geq 1 / k$ and for $t<1 / k$

$$
L_{k}(t)=-\int_{t}^{1 / k} L_{k-1}\left(\frac{x}{1-x}\right) \frac{d x}{x} .
$$

We may check easily that

$$
L_{k}(\alpha)=\frac{(-1)^{k}}{k !} I_{k}(1 / \alpha)
$$

The author was supported in part by NSF grant DMS-0500711. 
Broadhurst conjectures that there exists a sequence of constants $C_{k}$ (for integers $k \geq 0$ ) such that, as $t \rightarrow 0$ we have

$$
L_{k}(t)=\sum_{j=0}^{k} C_{j} \frac{(\log t)^{k-j}}{(k-j) !}+o(1) .
$$

Equivalently, in terms of $I_{k}(u)$, Broadhurst's conjecture reads, as $u \rightarrow \infty$,

$$
I_{k}(u)=\sum_{j=0}^{k}\left(\begin{array}{l}
k \\
j
\end{array}\right) D_{j}(\log u)^{k-j}+o(1),
$$

with $D_{j}=(-1)^{j} j ! C_{j}$. He found that $C_{0}=1, C_{1}=0, C_{2}=-\pi^{2} / 12$, $C_{3}=-\zeta(3) / 3$, and formulated the elegant conjecture that

$$
\sum_{k=0}^{\infty} C_{k} z^{k}=\frac{e^{\gamma z}}{\Gamma(1-z)}
$$

We establish this conjecture below.

Proposition 1. For natural numbers $k \geq 0$ define the constants

$$
C_{k}=\frac{1}{k !} \frac{1}{2 \pi i} \int_{c-i \infty}^{c+i \infty} \frac{e^{s}}{s}(\log s+\gamma)^{k} d s,
$$

where $c>0$ and the integral converges conditionally and is interpreted as $\lim _{T \rightarrow \infty} \int_{c-i T}^{c+i T}$. We have, for all complex $z$,

$$
\sum_{k=0}^{\infty} C_{k} z^{k}=\frac{e^{\gamma z}}{\Gamma(1-z)}
$$

Proof. First we rewrite the definition of $C_{k}$ by integrating by parts. Thus

$$
C_{k}=\frac{1}{k !} \frac{1}{2 \pi i} \int_{c-i \infty}^{c+i \infty} e^{s}\left(\frac{(\log s+\gamma)^{k}}{s^{2}}-\frac{k(\log s+\gamma)^{k-1}}{s^{2}}\right) d s .
$$

This integral is now absolutely convergent. Moreover, choosing $c=1$, we obtain the bound

$$
\left|C_{k}\right| \leq \frac{1}{2 \pi k !} \int_{-\infty}^{\infty} \frac{e(1+k)(\log (1+|t|)+\gamma+\pi / 2)^{k}}{1+t^{2}} d t \leq C(k+1)
$$

for some constant $C$. Thus the series $\sum_{k=0}^{\infty} C_{k} z^{k}$ converges absolutely for $|z|<1$ and defines in that region an analytic function.

Using our expression (11) we obtain that for $|z|<1$

$$
\sum_{k=0}^{\infty} C_{k} z^{k}=\frac{1}{2 \pi i} \int_{c-i \infty}^{c+i \infty} e^{s} \frac{e^{\gamma z} s^{z}}{s^{2}}(1-z) d s=\frac{e^{\gamma z}(1-z)}{\Gamma(2-z)}=\frac{e^{\gamma z}}{\Gamma(1-z)} .
$$

The relation connecting the contour integral to $1 / \Gamma(2-z)$ may be derived by deforming the line of integration to a contour traveling just above the real axis from $-\infty$ to zero, taking a detour around zero, and then traveling back to $-\infty$ just below the real axis. The resulting integral is Hankel's contour 
integral for the reciprocal of the $\Gamma$-function; see for example Chapter IX of [2], and in particular the "miscellaneous example" 24 which attributes our formula above to Laplace.

Since $e^{\gamma z} / \Gamma(1-z)$ is analytic for all $z \in \mathbb{C}$, we conclude that the series $\sum_{k=0}^{\infty} C_{k} z^{k}$ converges absolutely for all $z$ and equals $e^{\gamma z} / \Gamma(1-z)$.

Theorem 1. Setting $D_{j}=(-1)^{j} j ! C_{j}$ we have as $u \rightarrow \infty$

$$
I_{k}(u)=\sum_{j=0}^{k}\left(\begin{array}{l}
k \\
j
\end{array}\right) D_{j}(\log u)^{k-j}+O\left(\frac{(\log u)^{k}}{u}\right)
$$

and, equivalently

$$
L_{k}(t)=\sum_{j=0}^{k} C_{j} \frac{(\log t)^{k-j}}{(k-j) !}+O\left(t(|\log t|)^{k}\right) .
$$

Proof. Recall Perron's formula which gives, for any $c>0$, that

$$
\frac{1}{2 \pi i} \int_{c-i \infty}^{c+i \infty} \frac{e^{\lambda s}}{s} d s= \begin{cases}1 & \text { if } \lambda>0 \\ 0 & \text { if } \lambda<0\end{cases}
$$

and for completeness we note that the integral is $1 / 2$ if $\lambda=0$. Note that the integral above is only conditionally convergent and should be understood as $\lim _{T \rightarrow \infty} \int_{c-i T}^{c+i T}$. Therefore we find that

$$
I_{k}(u)=\frac{1}{2 \pi i} \int_{c-i \infty}^{c+i \infty} \frac{e^{s}}{s} \int_{t_{1}, \ldots, t_{k} \geq 1} \frac{e^{-\left(t_{1}+\ldots+t_{k}\right) s / u}}{t_{1} \cdots t_{k}} d t_{1} \cdots d t_{k} d s .
$$

Making a change of variables in the inner integrals we obtain that

$$
I_{k}(u)=\frac{1}{2 \pi i} \int_{c-i \infty}^{c+i \infty} \frac{e^{s}}{s}\left(\int_{1 / u}^{\infty} \frac{e^{-t s}}{t} d t\right)^{k} d s .
$$

Now observe that

$$
\int_{1 / u}^{\infty} \frac{e^{-t s}}{t} d t=-\int_{0}^{1} \frac{1-e^{-t s}}{t} d t+\int_{1}^{\infty} \frac{e^{-t s}}{t} d t+\int_{0}^{1 / u} \frac{1-e^{-t s}}{t} d t+\log u .
$$

For $s$ with positive real part we may see that

$$
\int_{0}^{1} \frac{1-e^{-t s}}{t} d t-\int_{1}^{\infty} \frac{e^{-t s}}{t} d t=\log s+\gamma
$$

and thus we conclude that

$$
\int_{1 / u}^{\infty} \frac{e^{-t s}}{t} d t=\log u-\log s-\gamma+\int_{0}^{1 / u} \frac{1-e^{-t s}}{t} d t .
$$

Write $G(u, s)=\int_{0}^{1 / u}\left(1-e^{-t s}\right) / t d t$. Using (3) in (2) we find that

$$
I_{k}(u)=\sum_{j=0}^{k}\left(\begin{array}{c}
k \\
j
\end{array}\right) \frac{1}{2 \pi i} \int_{c-i \infty}^{c+i \infty} \frac{e^{s}}{s} G(u, s)^{j}(\log u-\log s-\gamma)^{k-j} d s .
$$


Using the binomial theorem and our definition of the constants $C_{j}$ in Proposition 1 we see that the term $j=0$ equals

$$
\frac{1}{2 \pi i} \int_{c-i \infty}^{c+i \infty} \frac{e^{s}}{s}(\log u-\log s-\gamma)^{k} d s=\sum_{\ell=0}^{k}\left(\begin{array}{l}
k \\
\ell
\end{array}\right) D_{\ell}(\log u)^{k-\ell} .
$$

It remains finally to estimate the terms with $j \neq 0$. Integration by parts shows that the $j$-th term is

$$
-\frac{1}{2 \pi i} \int_{c-i \infty}^{c+i \infty} e^{s} \frac{d}{d s}\left(\frac{G(u, s)^{j}}{s}(\log u-\log s-\gamma)^{k-j}\right) d s .
$$

Since, for $\operatorname{Re}(s)>0$,

$$
|G(u, s)|=O\left(\int_{0}^{1 / u} \min \left(|s|, \frac{1}{t}\right) d t\right)=O(\log (1+|s| / u))
$$

and

$$
\left|\frac{d}{d s} G(u, s)\right|=\frac{\left|1-e^{-s / u}\right|}{|s|}=O\left(\min \left(\frac{1}{|s|}, \frac{1}{u}\right)\right),
$$

we may bound our integrand above by (choosing $c=1$ and writing $s=c+i t$ )

$$
\begin{aligned}
\frac{(1+\log u+\log (1+|t|))^{k-j}}{1+|t|} & (\log (1+(1+|t|) / u))^{j-1} \min \left(\frac{1}{1+|t|}, \frac{1}{u}\right) \\
& \left.+\frac{(\log (1+(1+|t|) / u))^{j}}{1+|t|}\right) .
\end{aligned}
$$

Integrating this over $t$ from $-\infty$ to $\infty$ we conclude that the $j$-th term is $O\left((\log u)^{k} / u\right)$ as desired. This proves our Theorem.

We take this opportunity to make an historical observation on the Dickman function. While Dickman's paper [3] appears to be the first published account of the $\rho$-function, during the Focused Week on Quadratic Forms and Theta Functions held in March 2010 at the University of Florida, I chanced upon the following entry in Ramanujan's unpublished papers: see page 337 of [4]. We quote the second half of this page:

$\phi(x)$ is the no. of nos of the form

$$
2^{a_{2}} \cdot 3^{a_{3}} \cdot 5^{a_{5}} \cdots p^{a_{p}} \quad p \leq x^{\epsilon}
$$

not exceeding $x$.

$$
\begin{gathered}
\frac{1}{2} \leq \epsilon \leq 1, \quad \phi(x) \sim x\left\{1-\int_{\epsilon}^{1} \frac{d \lambda_{0}}{\lambda_{0}}\right\} \\
\frac{1}{3} \leq \epsilon \leq \frac{1}{2}, \quad \phi(x) \sim x\left\{1-\int_{\epsilon}^{1} \frac{d \lambda_{0}}{\lambda_{0}}+\int_{\epsilon}^{\frac{1}{2}} \frac{d \lambda_{1}}{\lambda_{1}} \int_{\lambda_{1}}^{1-\lambda_{1}} \frac{d \lambda_{0}}{\lambda_{0}}\right\}
\end{gathered}
$$




$$
\begin{aligned}
& \frac{1}{4} \leq \epsilon \leq \frac{1}{3}, \quad \phi(x) \sim x\left\{1 \quad-\int_{\epsilon}^{1} \frac{d \lambda_{0}}{\lambda_{0}}+\int_{\epsilon}^{\frac{1}{2}} \frac{d \lambda_{1}}{\lambda_{1}} \int_{\lambda_{1}}^{1-\lambda_{1}} \frac{d \lambda_{0}}{\lambda_{0}}\right. \\
&\left.-\int_{\epsilon}^{\frac{1}{3}} \frac{d \lambda_{2}}{\lambda_{2}} \int_{\lambda_{2}}^{\frac{1-\lambda_{2}}{2}} \frac{d \lambda_{1}}{\lambda_{1}} \int_{\lambda_{1}}^{1-\lambda_{1}} \frac{d \lambda_{0}}{\lambda_{0}}\right\} \\
& \frac{1}{5} \leq \epsilon \leq \frac{1}{4}, \quad \phi(x) \sim x \quad\left\{1-\int_{\epsilon}^{1} \frac{d \lambda_{0}}{\lambda_{0}}+\int_{\epsilon}^{\frac{1}{2}} \frac{d \lambda_{1}}{\lambda_{1}} \int_{\lambda_{1}}^{1-\lambda_{1}} \frac{d \lambda_{0}}{\lambda_{0}}\right. \\
&-\int_{\epsilon}^{\frac{1}{3}} \frac{d \lambda_{2}}{\lambda_{2}} \int_{\lambda_{2}}^{\frac{1-\lambda_{2}}{2}} \frac{d \lambda_{1}}{\lambda_{1}} \int_{\lambda_{1}}^{1-\lambda_{1}} \frac{d \lambda_{0}}{\lambda_{0}} \\
&\left.+\int_{\epsilon}^{\frac{1}{4}} \frac{d \lambda_{3}}{\lambda_{3}} \int_{\lambda_{3}}^{\frac{1-\lambda_{3}}{3}} \frac{d \lambda_{2}}{\lambda_{2}} \int_{\lambda_{2}}^{\frac{1-\lambda_{2}}{2}} \frac{d \lambda_{1}}{\lambda_{1}} \int_{\lambda_{1}}^{1-\lambda_{1}} \frac{d \lambda_{0}}{\lambda_{0}}\right\}
\end{aligned}
$$

and so on.

Digressing from the topic of this note, we point out that the first half of this page of Ramanujan is also of interest to number theorists. Here Ramanujan observes that for fixed $k$ the number of integers below $x$ with at most $k$ prime factors is asymptotically

$$
\frac{x}{\log x}\left(1+\log \log x+\frac{(\log \log x)^{2}}{2 !}+\ldots+\frac{(\log \log x)^{k-1}}{(k-1) !}\right) .
$$

He notes that this formula also holds "when $k$ is infinite." Ramanujan then asks whether the formula holds "when $k$ is a function of $x$ ". This interesting question on the uniformity with which such an asymptotic formula holds was settled by the work of Sathe [5] and Selberg [6].

Acknowledgments. I am grateful to Jeff Lagarias for drawing my attention to this problem.

\section{REFERENCES}

[1] D. Broadhurst, Dickman polylogarithms and their constants, preprint, arXiv: $1004.0519 \mathrm{v} 1$.

[2] E. T. Copson, An introduction to the theory of functions of a complex variable, Oxford University Press (1970).

[3] K. Dickman, On the frequency of numbers containing prime factors of a certain relative magnitude, Arkiv Mat. Astron. Fys. 22 (1930) 1-14.

[4] S. Ramanujan, The lost notebook and other unpublished papers, Narosa Publishing House, (1988).

[5] L. G. Sathe, On a problem of Hardy on the distribution of integers having a given number of prime factors. III and IV. J. Indian Math. Soc., 18 (1954), 27-42, and $43-81$.

[6] A. Selberg, Note on a paper by L. G. Sathe, J. Indian Math. Soc., 18 (1954), 83-87.

Department of Mathematics, Stanford University, Stanford, CA 94305, USA E-mail: ksound@math.stanford.edu 